\title{
Incidencia, causas y pronóstico del síncope en la población general
}

Incidence and Prognosis of Syncope. Soteriades S, Evans JC, Larson MG y cols. N Engl J Med 2002;347:878-85

\section{Objetivo}

Evaluar la epidemiología y el pronóstico del síncope en la población general.

\section{Diseño}

Framingham Heart Study. Estudio de cohorte prospectivo.

\section{Lugar}

Framingham, EE.UU.

\section{Pacientes}

Se siguieron 7814 sujetos de la población general por un promedio de 17 años entre los años 1971 y 1998.

Se incluyeron solo los casos inequívocos de síncope basándose en la historia clínica y en el consenso de un comité médico. La causa era asignada a través del diagnóstico clínico de un internista y un cardiólogo ciegos a los resultados. Se evaluaron cuatro categorías etiológicas: causas cardíacas (isquemia y arritmias), causa desconocida, causa neurológica (accidente cerebrovascular [ACV], ataque isquémico transitorio y epilepsia) y causa vasovagal u otra (que incluía también la ortostática, la secundaria a medicación y otras).

\section{Medición de Resultados}

Se evaluó la incidencia, la recurrencia y las causas de síncope en relación a tres resultados principales: muerte de cualquier causa muerte coronaria o infarto de miocardio (IAM); y ACV fatal y no fatal. Se realizaron análisis multivariables para evaluar la contribución específica de cada categoría etiolgica en estos resultados, ajustando por edad, sexo, historia de enfermedad cardio vascular, tabaquismo, hipertensión, diabetes, colesterol, frecuencia cardica y uso de medicación cardiovascular.

Resultados

La edad media de los 7814 participantes fue de 51.14 años (DS
14.4). El $46 \%$ fueron hombres. De los 822 pacientes que presentaron síncope, se incluyeron 727 (los otros lo presentaron en el último examen y no tenían seguimiento). La incidencia del mismo fue de 6.2 cada 1000 pacientes/año, y fue mayor luego de los 70 años (entre 11 a 19 cada 1000 pacientes/año) y en los pacientes con enfermedad cardiovascular (10.6 cada 1000 pacientes/año). Las causas más frecuentes fueron la vasovagal $(21.2 \%)$, cardíaca $(9.5 \%)$, y ortostática $(9.4 \%)$. En el $36.6 \%$ de los casos la causa fue desconocida.

\begin{tabular}{|c|c|c|c|c|}
\hline \multirow[t]{2}{*}{ Evento } & $\begin{array}{l}\text { Cualquier } \\
\text { Síncope }\end{array}$ & $\begin{array}{l}\text { Síneope } \\
\text { Cardíaco }\end{array}$ & $\begin{array}{c}\text { Causa } \\
\text { Desconocida }\end{array}$ & $\begin{array}{c}\text { Causa } \\
\text { Neurológica }\end{array}$ \\
\hline & \multicolumn{4}{|c|}{ Riesgo Relativo Ajustado (I695\%) } \\
\hline Muerte por cualquier caus & $\begin{array}{c}1.31 \\
(1.14 \text { a } 1.51)^{8}\end{array}$ & $\begin{array}{c}2.01 \\
(1.48 \text { a } 2.73)^{8}\end{array}$ & $\begin{array}{c}1.32 \\
(1.09 \mathrm{a} 1.60)^{8}\end{array}$ & $\begin{array}{c}1.54 \\
(1.21 \mathrm{a} 2.12)^{8}\end{array}$ \\
\hline IAM 0 & $\begin{array}{c}1.27 \\
(0.99 \text { a } 1.64)\end{array}$ & $\begin{array}{c}2.66 \\
(1.69 \text { a } 4.19)^{8}\end{array}$ & $\begin{array}{c}1.31 \\
(0.92 \text { a } 1.86)\end{array}$ & $\begin{array}{c}0.79 \\
(0.37 \text { a } 1.69)\end{array}$ \\
\hline CV fatal o no fatal & $\begin{array}{c}1.06 \\
(0.77 \text { a } 1.45)\end{array}$ & $\begin{array}{c}2.01 \\
(1.06 \text { a } 3.80)^{8}\end{array}$ & $\begin{array}{c}0.66 \\
(0.39 \text { a } 1.11)\end{array}$ & $\begin{array}{c}2.96 \\
(1.69 \mathrm{a} 5.18)^{8}\end{array}$ \\
\hline
\end{tabular}

$\& p>0.05$

La causa vasovagal (que incluía también la ortostática y la debido a drogas) no implicó un mayor riesgo de morbimortalidad cardiovascular. Solo el 21.6 de los pacientes tuvieron recurrencias (la gran mayoría una sola); el riesgo fue especialmente alto en los pacientes con causa cardíaca.

\section{Conclusión}

Las personas con síncope cardíaco tienen un riesgo amentado de muerte por cualquier causa y de eventos cardiovasculares, y las personas con síncope de causa desconocida tienen una mayor mortalidad. Los síncopes vasovagales son de buen pronóstico.

Fuente de financiamiento: National Heart, Lung and Blood Institute, EE.UU.

\section{Comentario}

Estamos ante la presencia de un nuevo reporte del estudio Framingham, el paradigma de estudio de cohorte que acuñó un término hoy tan importante como "factor de riesgo". En este caso Soteriades y cols. se dedicaron a estudiar el síncope (pérdida súbita de conocimiento con pérdida del tono postural y recuperación espontánea), sus causas y su pronóstico.

La gran ventaja de este estudio es que refleja al síncope en la población general con un extenso tiempo de seguimiento; muchos de los estudios previos fueron hechos en guardias, salas de hospital o centros de referencia. ${ }^{1-6}$ Varias son sus puntos a señalar: 1) La incidencia de síncope fue similar en hombres y en mujeres, aumentó claramente con la edad, y fue del doble en gente con historia de enfermedad cardiovascular. 2) El síncope cardíaco duplicó el riesgo de muerte y también aumentó el riesgo de IAM y eventos cardiovasculares no fatales. Esto se mantuvo incluso en pacientes sin enfermedad cardíaca previa. 3) El síncope neurológico se asoció con un aumento intermedio del riesgo de muerte e incrementó el de ACV (y el resultado se mantuvo al incluir o excluir síncope convulsivo). 4) La causa más frecuentemente identificada, el síncope vasovagal y sus "parientes" por hipotensión ortostática y relacionado a drogas no se asoció a un peor pronóstico. 5) El haber tenido síncope de causa desconocida confirió un $30 \%$ de aumento de riesgo de mortalidad, debido principalmente a muerte por IAM o coronaria.

Un punto importante a señalar es que este fue un estudio observacional, no diseñado específicamente para evaluar profundamente las causas y el pronóstico del síncope. Aunque puede haber habido algunos sesgos de recuerdo y de mala clasificación, * sus resultados son bastante coincidentes con la literatura previa. Es probable que a la primera razón se deba el hallazgo del aumento de riesgo en el síncope de causa desconocida. En estudios diseñados especialmente para evaluar la etiología del síncope, muchos pacientes diagnosticados inicialmente como de causa desconocida eran luego diagnosticados como de causa cardíaca al estudiarlos mejor. Es por ello que probablemente los pacientes con síncope de causa desconocida en este estudio eran una mezcla de dos poblaciones: aquellos con síncope cardíaco no diagnosticado (con el consiguiente aumento de riesgo); y aquellos sin una causa cardíaca y con un buen pronóstico.

Conclusiónes del Comentador: Este estudio en población general confirma el hallazgo de estudios previos como el mayor riesgo que implica el síncope cardíaco y el buen pronóstico de vasovagal?. Por otro lado, sugiere que el síncope de causa desconocida incluiría a pacientes con aumento de riesgo de muerte, por lo que se debería pensar en estudiarlos más detalladamente en busca de causas cardíacas no detectadas inicialmente.

\section{Dr. Federico Augustovski [ Unidad de Medicina Familiar y Preventiva. Hospital Italiano de Buenos Aires ]}

\section{Referencias}

1.Kapoor WN, Karpf M, Wieand S, Peterson JR, Levey GS. A prospective evaluation and follow-up of patients with syncope. N Engl J Med 1983;309:197-204.

2.Day SC, Cook EF, Funkenstein H, Goldman L. Evaluation and outcome of emergency room patients with transient loss of consciousness. Am J Med 1982;73:15-23.

3. Eagle KA, Black HR, Cook EF, Goldman L. Evaluation of prognostic classifications for patients with syncope. Am J Med 1985;79:455-460

4.Martin TP, Hanusa BH, Kapoor WN. Risk stratification of patients with syncope. Ann Emerg Med 1997;29:459-466.

5.Silverstein MD, Singer DE, Mulley AG, Thibault GE, Barnett GO. Patients with syncope admitted to medical intensive care units. JAMA 1982;248:1185-1189. 6.Kapoor WN. Evaluation and outcome of patients with syncope. Medicine (Baltimore) 1990;69:160-175

7.Augustovski F. Síncope. En: Profam: Programa de Educación a Distancia de Medicina Familiar y Ambulatoria / Esteban Rubinstein; Miguel Zárate, Paula Carrete, editores. -- 2ª ed.

-- Buenos Aires: Fundación MF "Para el Desarrollo de la Medicina Familiar y la Atención primaria de la Salud", 2001. 